\title{
Pulmonary Vein Isolation for Vagotonic, Adrenergic, and Random Episodes of Paroxysmal Atrial Fibrillation
}

\author{
HAKAN ORAL, M.D., AMAN CHUGH, M.D., CHRISTOPH SCHARF, M.D., BURR HALL, M.D., \\ PETER CHEUNG, M.D., SRIKAR VEERAREDDY, M.D., GERALD F. DANESHVAR, B.S., \\ FRANK PELOSI JR., M.D., and FRED MORADY, M.D.
}

From the Division of Cardiology, University of Michigan, Ann Arbor, Michigan, USA

\begin{abstract}
Pulmonary Vein Isolation. Introduction: Based on the clinical history, paroxysmal atrial fibrillation (PAF) may be classified as vagotonic, adrenergic, or random. It is unclear whether pulmonary vein (PV) isolation is equally effective for these types of PAF.

Methods and Results: Segmental ostial ablation to isolate the PVs was performed in 188 consecutive patients (mean age $53 \pm 12$ years) with PAF. Based on the clinical history, PAF was classified as random in 136 patients $(72 \%)$, adrenergic in $30(16 \%)$, and vagotonic in $22(12 \%)$. Three or four PVs were targeted for isolation in all patients, and successful isolation was achieved in $96 \%$ of targeted PVs. At 1-year follow-up, $69 \%$ of patients with random $\mathrm{AF}, 83 \%$ of patients with adrenergic $\mathrm{AF}$, and $50 \%$ of patients with vagotonic $A F$ were free from recurrent episodes of $\mathrm{AF}$ in the absence of any antiarrhythmic drug therapy. Vagotonic $A F$ was an independent clinical predictor of recurrent $\mathrm{AF}(\mathrm{P}=\mathbf{0 . 0 3})$.

Conclusion: PV isolation has a lower efficacy in patients with vagotonic PAF than in patients with adrenergic or random episodes of PAF, suggesting that the PVs less often play an important role in vagotonic PAF. (J Cardiovasc Electrophysiol, Vol. 15, pp. 402-405, April 2004)
\end{abstract}

atrial fibrillation, catheter ablation, pulmonary vein, vagotonic atrial fibrillation

\section{Introduction}

In some patients, paroxysmal atrial fibrillation (PAF) may occur during states of increased adrenergic activity, whereas in others, PAF may occur during states of heightened vagal tone. ${ }^{1-4}$ Based on the different autonomic milieu in which they occur, adrenergic and vagotonic PAF may have different mechanisms. Although pulmonary vein (PV) isolation often is effective in eliminating PAF, prior studies have not distinguished subgroups of PAF ${ }^{5,6}$ Therefore, it is unclear whether PV isolation has similar efficacy in patients with vagotonic, adrenergic, and random episodes of PAF. The aim of this study was to compare the long-term outcomes after PV isolation in patients with vagotonic, adrenergic, and random episodes of PAF.

\section{Methods}

\section{Study Subjects}

PV isolation was performed in 188 consecutive patients with symptomatic, drug-refractory PAF. The clinical characteristics of the patients are given in Table 1.

Supported by the Ellen and Robert Thompson Atrial Fibrillation Research Fund.

Address for correspondence: Hakan Oral, M.D., Division of Cardiology, TC B1 140D, University of Michigan, 1500 E. Medical Center Drive, Ann Arbor, MI 48109-0311. Fax: 734-936 7026; E-mail: oralh@umich.edu

Manuscript received 12 August 2003; Accepted for publication 9 December 2003.

doi: 10.1046/j.1540-8167.2004.03432.x

\section{Classification of PAF}

Coumel et al. ${ }^{1,7}$ first described the syndromes of vagotonic and adrenergic PAF. In his studies, Coumel indicated that these types of PAF can be reliably identified based on details of the clinical history; therefore, classification of PAF in the present study was based on a detailed clinical history. Patients with PAF that occurred at least $90 \%$ of the time during sleep or after dinner while patients were relaxing were classified as having vagotonic PAF. Patients with PAF that occurred at least $90 \%$ of the time during or shortly after strenuous exertion or other states of adrenergic activation were classified as having adrenergic PAF. Other patients in whom episodes occurred predominantly on a random basis were described as having random episodes of $\mathrm{PAF}^{2-4}$

\section{PV Isolation}

All patients provided written informed consent. Electrical isolation of the PVs was performed by segmental ostial applications of radiofrequency energy, as described previously. ${ }^{5,6,8}$ The left superior, left inferior, and right superior PVs were targeted in all 188 patients. The right inferior PV also was targeted in 77 patients (41\%). The percentage of patients who underwent isolation of the right inferior PV did not differ among the three subgroups $(\mathrm{P}=0.8)$. Complete isolation was achieved in $96 \%$ of targeted PVs, with no differences between patients with vagotonic, adrenergic, and random episodes of PAF.

After the ablation procedure, all patients underwent continuous monitoring for 24 hours. Heparin was administered intravenously for 24 hours and was followed by lowmolecular-weight heparin for 4 days and warfarin for 1 to 3 months after the procedure. 


\begin{tabular}{lcccc}
\hline \multicolumn{5}{c}{ TABLE 1 } \\
\multicolumn{5}{c}{ Clinical Characteristics of Patients with Vagotonic, Adrenergic, and } \\
& Random PAF & & \\
\hline & Vagotonic & Adrenergic & Random & \\
& PAF & PAF & PAF & P Value \\
\hline N & 22 & 30 & 136 & \\
Age (years) & $55 \pm 10$ & $50 \pm 12$ & $53 \pm 12$ & 0.3 \\
Gender (male/female) & $19 / 3$ & $27 / 3$ & $107 / 29$ & 0.3 \\
Duration of PAF (years) & $6 \pm 5$ & $6 \pm 7$ & $8 \pm 8$ & 0.3 \\
Episodes per month & $12 \pm 11$ & $16 \pm 14$ & $17 \pm 17$ & 0.5 \\
LVEF & $0.54 \pm 0.05$ & $0.55 \pm 0.04$ & $0.55 \pm 0.04$ & 0.4 \\
Left atrial size (mm) & $42 \pm 6$ & $39 \pm 4$ & $39 \pm 5$ & 0.2 \\
Structural heart disease & $4(18 \%)$ & $5(17 \%)$ & $13(10 \%)$ & 0.3 \\
\hline Conting
\end{tabular}

Continuous variables are expressed as mean \pm SD.

\section{Follow-Up}

All patients were seen in an outpatient clinic 4 to 6 weeks and every 3 to 6 months after the ablation procedure. Whenever they reported symptoms, they were provided with an event recorder to document the cause of symptoms. Because episodes of PAF that occur within 4 weeks after PV isolation may be transient, episodes of PAF that were limited to the first month of follow-up were excluded from the analysis. ${ }^{9}$ During a mean duration of $445 \pm 190$ days, no patient was lost to follow-up.

\section{Statistical Analysis}

Continuous variables are expressed as mean $\pm 1 \mathrm{SD}$ and were compared by Student's $t$-test. Categorical variables were compared by Chi-square analysis or with Fisher's exact test. Differences between continuous variables were determined by analysis of variance. Kaplan-Meier analysis with the log rank test was used to determine the probability of freedom from recurrent PAF after PV isolation. Cox multivariate regression analyses were performed to determine the clinical predictors of freedom from PAF. P $<0.05$ was considered statistically significant.

\section{Results}

\section{Modes of Onset of AF and Clinical Characteristics}

Among the 188 patients, 22 (12\%) were classified as having vagotonic PAF, 30 (16\%) were classified as having adrenergic PAF, and the remaining 136 patients (72\%) were classified as having random episodes of PAF.

There were no significant differences with regard to age, gender, duration of PAF, frequency of PAF, left ventricular ejection fraction, left atrial size, or presence of structural heart disease among the three subgroups of PAF (Table 1).

\section{Freedom from Recurrent PAF}

At 1-year follow-up, PV isolation resulted in freedom from recurrent PAF in 50\% of patients with vagotonic PAF (Fig. 1). The percentage of patients free of recurrent PAF after PV isolation was higher among patients with adrenergic PAF $(83 \%, \mathrm{P}=0.02)$ and among patients with a history of random episodes of PAF $(69 \%, \mathrm{P}=0.05)$ compared to those with vagotonic PAF. The percentage of patients free of PAF at

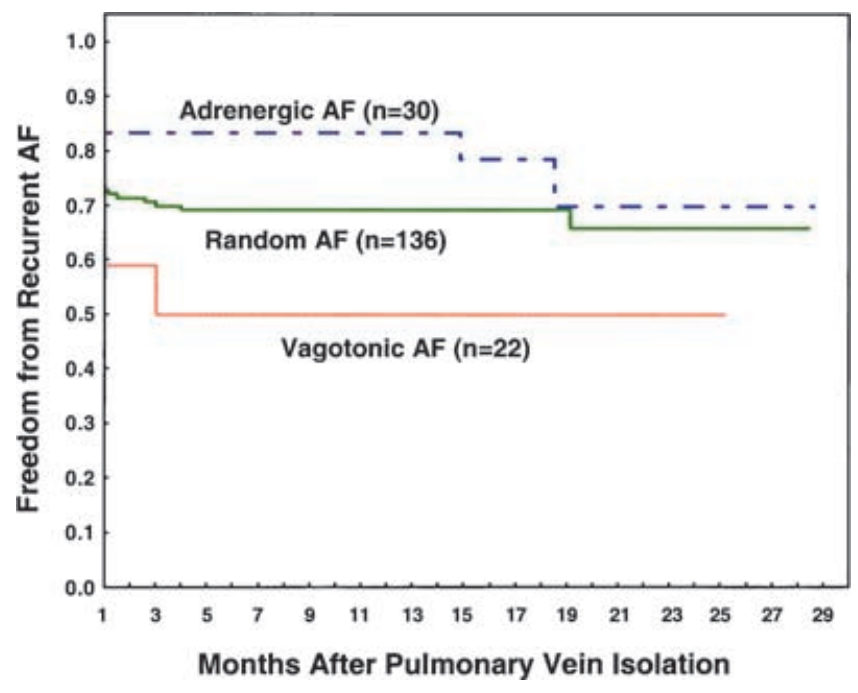

Figure 1. Freedom from paroxysmal atrial fibrillation $(P A F)$ in patients with vagotonic, adrenergic, and random PAF. The recurrences of $P A F$ that occurred only within the first month of follow-up were blanked out. $P=0.04$ for vagotonic vs adrenergic $A F ; P=0.07$ for vagotonic vs random $A F ; P=$ 0.3 for random vs adrenergic AF (log rank test).

1-year follow-up did not differ significantly between patients with adrenergic and random episodes of $\operatorname{PAF}(\mathrm{P}=0.3)$.

\section{Multivariate Analysis of Predictors of Outcome}

Among the clinical variables of age, gender, duration of PAF, frequency of PAF, left ventricular ejection fraction, left atrial size, presence of structural heart disease, and whether or not the PAF was vagotonic, the only independent predictor of recurrent episodes of PAF after PV isolation was vagotonic $\mathrm{PAF}(\mathrm{P}=0.03)$. $\mathrm{P}$ values for the univariate and multivariate analyses are listed in Table 2.

\section{Discussion}

\section{Main Findings}

The clinical syndromes of vagotonic and adrenergic PAF were first described in anecdotal fashion 25 years ago, ${ }^{1}$ but the prevalence of these types of PAF has never been defined. In the present study of patients with drug-refractory PAF referred for catheter ablation, the clinical history was

\section{TABLE 2}

$\mathrm{P}$ value for the Univariate and Multivariate Analyses of Clinical Predictors of Recurrence of AF After PV isolation

\begin{tabular}{lcc}
\hline & $\begin{array}{c}\text { P Value for } \\
\text { Univariate } \\
\text { Analysis }\end{array}$ & $\begin{array}{c}\text { P Value for } \\
\text { Multivariate } \\
\text { Analysis }\end{array}$ \\
\hline Age (years) & 0.1 & 0.4 \\
Gender (male/female) & 0.7 & 0.6 \\
Duration of PAF (years) & 0.02 & 0.05 \\
Frequency of episodes of AF & 0.5 & 0.7 \\
LVEF & 0.4 & 0.4 \\
Left atrial size (mm) & 0.9 & 0.5 \\
Structural heart disease & 0.9 & 0.7 \\
Vagotonic AF & 0.04 & 0.03 \\
\hline
\end{tabular}

$\overline{\mathrm{LVEF}}=$ left ventricular ejection fraction; $\mathrm{PAF}=$ paroxysmal atrial fibrillation. 
characteristic of vagotonic and adrenergic PAF in $12 \%$ and $16 \%$ of patients, respectively.

PV isolation was found to be significantly less effective among the patients with vagotonic PAF than among the patients with adrenergic or random episodes of PAF. This observation implies that the PVs less often serve as a key source of triggers or drivers of atrial fibrillation in vagotonic PAF than in other types of PAF.

\section{Vagotonic PAF}

An increase in vagal tone results in shortening of the atrial effective refractory period, an increase in dispersion of refractoriness, and an increase in the vulnerability to $\mathrm{AF}^{8,10,11}$ In addition, vagal stimulation may increase the frequency of premature atrial depolarizations from anywhere in the atria. ${ }^{8,10,11}$ These effects of heightened vagal tone may explain why PV isolation is less often effective in eliminating the vagotonic variety of PAF than other types of PAF. However, the fact that PV isolation was still effective in $\sim 50 \%$ of patients with vagotonic AF suggests that the PVs play a role in at least some patients with vagotonic AF. It also is possible that segmental ostial ablation has an effect on autonomic innervation of the left atrium. In any case, to improve the efficacy of the procedure in patients with vagotonic AF, it may be appropriate to consider ablation of non-PV triggers and/or left atrial substrate modification in addition to PV isolation.

\section{Adrenergic PAF}

Prior experimental and clinical studies have suggested that the vein of Marshall may play an important role in the initiation of adrenergically mediated episodes of atrial fibrillation. ${ }^{12,13}$ In the present study, patients with adrenergic PAF responded favorably to PV isolation, without any attempts to identify or ablate the vein of Marshall. However, because the vein of Marshall may be located close to the left superior $\mathrm{PV},{ }^{12}$ it is possible that some applications of radiofrequency energy aimed at the ostium unintentionally also ablated the insertion of the vein of Marshall. It also is possible that both the PVs and the vein of Marshall play a role in generating adrenergic PAF, but that isolation of the PVs by itself often is sufficient to eliminate this type of PAF.

\section{Clinical Predictors of Recurrent AF After PV Isolation}

Vagotonic AF was the only independent predictor of recurrent episodes of AF among the clinical variables of age, gender, duration of PAF, frequency of PAF, left ventricular ejection fraction, left atrial size, presence of structural heart disease, and whether or not the PAF was vagotonic. Although the duration of $\mathrm{AF}$ also was a univariate predictor, in multivariate analysis, vagotonic AF emerged as a stronger independent predictor. As reported previously, ${ }^{14}$ the duration of AF may be one of the independent predictors for recurrences of AF. However, in that study, vagotonic AF was not assessed and was not incorporated into the analysis as a clinical variable. The amount of left atrial electroanatomic remodeling may increase with the duration of AF, and this may decrease the relative importance of the PVs in the generation of AF. However, in patients with vagotonic AF, the PVs may not be as critical in the initiation and perpetuation of AF as in other types of AF. Therefore, when vagotonic AF is also included in a multivariate analysis, it may become more prominent than the duration of AF, because vagotonic AF may indicate the presence of a non-PV substrate for the AF.

\section{Study Limitations}

A limitation of this study is that there were no attempts to identify asymptomatic episodes of PAF. Therefore, it is possible that the efficacy of PV isolation was overestimated. However, there would be no reason for asymptomatic PAF to be less likely in patients with vagotonic PAF than in patients with adrenergic or random PAF. Therefore, the lower efficacy of PV isolation among patients with vagotonic PAF in all likelihood still would be a valid finding.

In some patients, most but not all episodes of PAF occurred during characteristic periods of heightened vagal or adrenergic tone. Allowing for the possibility of occasional random episodes of PAF in patients with vagotonic or adrenergic PAF, patients were included in the vagotonic and adrenergic subgroups if at least $90 \%$ of their episodes were consistent with that type of PAF. This $90 \%$ cutoff was arbitrarily chosen and was dependent on self-assessment by the patients. Detailed $\operatorname{logs}$ of all episodes were not available.

The patients in this study were selected to undergo catheter ablation because they had frequent, symptomatic, drugrefractory episodes of PAF. The prevalence of vagotonic and adrenergic PAF may be different in an unselected population of patients with PAF.

\section{Conclusion}

In approximately $25 \%$ of patients with PAF, the clinical history may be consistent with vagotonic or adrenergic PAF. Identification of these subgroups of patients with PAF has therapeutic implications. For example, in patients with vagotonic PAF, drugs that increase vagal tone, e.g., digitalis, may make episodes of PAF more frequent. In contrast, among patients with adrenergic PAF, beta-blockers, which usually provide only control of the ventricular rate, may prevent episodes of PAF.

The results of the present study suggest that identification of vagotonic PAF has another therapeutic implication. Whereas approximately $70 \%$ or more of patients with adrenergic or random episodes of PAF have an excellent outcome 1 year after PV isolation, this is the case in only $50 \%$ of patients with vagotonic PAF. Therefore, among potential candidates for PV isolation, if the clinical history indicates that a patient has vagotonic PAF, it may be appropriate to consider an alternative ablation strategy. ${ }^{14}$

\section{References}

1. Coumel P, Attuel P, Lavallee J, Flammang D, Leclercq JF, Slama R: [The atrial arrhythmia syndrome of vagal origin]. Arch Mal Coeur Vaiss 1978;71:645-656.

2. Coumel P: Autonomic influences in atrial tachyarrhythmias. J Cardiovasc Electrophysiol 1996;7:999-1007.

3. Chen YJ, Chen SA, Tai CT, Wen ZC, Feng AN, Ding YA, Chang MS: Role of atrial electrophysiology and autonomic nervous system in patients with supraventricular tachycardia and paroxysmal atrial fibrillation. J Am Coll Cardiol 1998;32:732-738.

4. Schauerte P, Scherlag BJ, Pitha J, Scherlag MA, Reynolds D, Lazzara R, Jackman WM: Catheter ablation of cardiac autonomic nerves for prevention of vagal atrial fibrillation. Circulation 2000;102:2774-2780.

5. Haissaguerre M, Shah DC, Jais P, Hocini M, Yamane T, Deisenhofer I, Chauvin M, Garrigue S, Clementy J: Electrophysiological breakthroughs from the left atrium to the pulmonary veins. Circulation 2000;102:2463-2465. 
6. Oral H, Knight BP, Tada H, Ozaydin M, Chugh A, Hassan S, Scharf C, Lai SW, Greenstein R, Pelosi F Jr, Strickberger SA, Morady F: Pulmonary vein isolation for paroxysmal and persistent atrial fibrillation. Circulation 2002;105:1077-1081.

7. Coumel P: Cardiac arrhythmias and the autonomic nervous system. J Cardiovasc Electrophysiol 1993;4:338-355.

8. Kneller J, Zou R, Vigmond EJ, Wang Z, Leon LJ, Nattel S: Cholinergic atrial fibrillation in a computer model of a two- dimensional sheet of canine atrial cells with realistic ionic properties. Circ Res 2002;90:E73E87.

9. Oral H, Knight BP, Ozaydin M, Tada H, Chugh A, Hassan S, Scharf C, Lai SW, Greenstein R, Pelosi F, Strickberger SA, Morady F: Clinical significance of early recurrences of atrial fibrillation after pulmonary vein isolation. J Am Coll Cardiol 2002;40:100-104.

10. Hirose M, Carlson MD, Laurita KR: Cellular mechanisms of vagally mediated atrial tachyarrhythmia in isolated arterially perfused canine right atria. J Cardiovasc Electrophysiol 2002;13:918-926.
11. Sharifov OF, Zaitsev AV, Rosenshtraukh LV, Kaliadin AY, Beloshapko GG, Yushmanova AV, Schuessler RB, Boineau JP: Spatial distribution and frequency dependence of arrhythmogenic vagal effects in canine atria. J Cardiovasc Electrophysiol 2000;11:1029-1042.

12. Hwang C, Wu TJ, Doshi RN, Peter CT, Chen PS: Vein of Marshall cannulation for the analysis of electrical activity in patients with focal atrial fibrillation. Circulation 2000;101:1503-1505.

13. Wu TJ, Ong JJ, Chang CM, Doshi RN, Yashima M, Huang HL, Fishbein MC, Ting CT, Karagueuzian HS, Chen PS: Pulmonary veins and ligament of Marshall as sources of rapid activations in a canine model of sustained atrial fibrillation. Circulation 2001;103:1157-1163.

14. Pappone C, Oreto G, Rosanio S, Vicedomini G, Tocchi M, Gugliotta F, Salvati A, Dicandia C, Calabro MP, Mazzone P, Ficarra E, Di Gioia C, Gulletta S, Nardi S, Santinelli V, Benussi S, Alfieri O: Atrial electroanatomic remodeling after circumferential radiofrequency pulmonary vein ablation: efficacy of an anatomic approach in a large cohort of patients with atrial fibrillation. Circulation 2001;104:2539-2544. 